\title{
Application of 3D modeling and printing technology in accurate resection of complicated thoracic tumors
}

\author{
Deli Tan ${ }^{1,2 \#}$, Jie Yao ${ }^{1 \#}$, Xing $\mathrm{Hua}^{3}$, Jingyao $\mathrm{Li}^{2}$, Zhou $\mathrm{Xu}^{1}$, Yi Wu${ }^{1}$, Wei $\mathrm{Wu}^{2}$ \\ ${ }^{1}$ Institute of Digital Medicine, Biomedical Engineering College, ${ }^{2}$ Thoracic Surgery Department, Southwest Hospital, ${ }^{3}$ Ultrasound Department, \\ Southwest Hospital, Army Medical University (Third Military Medical University), Chongqing, China \\ Contributions: (I) Conception and design: Y Wu, W Wu; (II) Administrative support: D Tan, Y Wu, W Wu; (III) Provision of study materials or \\ patients: D Tan, W Wu; (IV) Collection and assembly of data: D Tan, J Yao, X Hua; (V) Data analysis and interpretation: J Yao, Z Xu, J Li; (VI) \\ Manuscript writing: All authors; (VII) Final approve of manuscript: All authors. \\ \#These authors contributed equally to this work. \\ Correspondence to: Yi Wu. Institute of Digital Medicine, Biomedical Engineering College, Army Medical University, Chongqing 400038, China. \\ Email: wuy1979@tmmu.edu.cn; Wei Wu. Department of Thoracic Surgery, Southwest Hospital, Army Medical University, No. 30 of Gaotanyan St., \\ Chongqing 400038, China. Email: wuweiyahoo@sohu.com.
}

Background: To explore the application value of three-dimensional (3D) reconstruction and 3D printing in preoperative evaluation of precise resection of complicated thoracic tumors.

Methods: A retrospective analysis of 34 patients with complicated thoracic tumors who were treated by radical surgery from March 2016 to June 2019 was made. According to whether 3D reconstruction and 3D printing was used, the patients were divided into research group and control group. In the control group, preoperative evaluation was performed according to CT image data, and the operation plan was drawn up; in the research group, preoperative simulation and preoperative operation plan design were carried out according to $3 \mathrm{D}$ reconstruction and $3 \mathrm{D}$ printing technology. The operation time, change of operation approach, intraoperative blood loss, hospitalization time and postoperative complications were compared between the two groups. We also retrospectively reviewed additional 12 cases of unresectable complicated thoracic tumors. The above 34 patients who were treated by radical surgery were set as the resectable group. Three-dimensional reconstruction was performed for all cases. The tumor size, location, smoothness of tumor-vascular contact surface, close contact with adjacent organs were compared between these two groups. Results: The 3D reconstruction and 3D printing model were successfully established. The indexes of operation time, change of incision approach and blood loss in the research group were lower than those in the control group $(\mathrm{P}<0.05)$. All the patients were followed up for 6 months, and there was no death, no tumor recurrence and metastasis in the two groups. In the unresectable group, the score of position and smoothness of tumor-vascular contact surface were significantly higher than that in the resectable group.

Conclusions: $3 \mathrm{D}$ reconstruction and $3 \mathrm{D}$ printing can effectively help surgeons carry out accurate surgical treatment, reduce the operation time and bleeding, reduce the risk of surgery, and facilitate the postoperative rehabilitation of patients, which has the value of promotion and application.

Keywords: Complicated thoracic tumor; chest wall; three-dimensional printing technology (3D printing technology); 3D reconstruction

Submitted Feb 20, 2020. Accepted for publication Sep 25, 2020.

doi: 10.21037/atm-20-1791

View this article at: http://dx.doi.org/10.21037/atm-20-1791 


\section{Introduction}

The complicated thoracic tumors refer to the tumors that are difficult for surgical treatment, including large tumors in the chest cavity, tumors with complicated spatial relationship, or tumors invading adjacent large blood vessels and important organs. Due to the complicated anatomy of thoracic tumor and its close relationship with the surrounding blood vessels and organs, it is difficult to recognize its three-dimensional (3D) structure and its spatial adjacent relationship in the two-dimensional images (1). Thus, surgical treatment is difficult, and there are many intra-operative and post-operative complications. Surgical treatment of complicated thoracic tumors remains to be a challenge for thoracic surgeons.

The 3D modeling and printing technology has been more frequently applied in medical treatment, which can visualize the abstract structures and simplify complex structures in an easy way. As a new evaluation method, it has been widely used in orthopedics, liver surgery, and neurology. Before surgery, the surgeons collect computed tomography (CT) or computed tomography angiography (CTA) image data of patients through $3 \mathrm{D}$ processing software, perform image segmentation and $3 \mathrm{D}$ reconstruction, obtain $3 \mathrm{D}$ reconstructed images, and apply 3D printers to print 3D models of human organs and abnormal lesions (2-9). It helps the surgeon to perform preoperative evaluation and simulation, determine an appropriate surgical plan, improve the efficacy of surgery, and reduce postoperative complications. However, there are relatively fewer application of $3 \mathrm{D}$ digital modeling and printing technology in complicated thoracic tumor surgery. In this study, $3 \mathrm{D}$ reconstruction and $3 \mathrm{D}$ printing technology were used to perform preoperative evaluation and surgical plan design on 34 patients with complicated thoracic tumors. By grouping surgeries, intraoperative and postoperative complications were analyzed to explore the application value of this technology in preoperative evaluation of complicated thoracic tumors. We present the following article in accordance with the STROBE reporting checklist (available at http://dx.doi. org/10.21037/atm-20-1791).

\section{Methods}

\section{Patient selection criteria}

Inclusion criteria included: (I) any thoracic tumor which was diagnosed according to clinical symptoms, imaging data, and puncture biopsy before operation; (II) no distant metastasis occurred before surgery which was excluded by positron emission tomography (PET) scan or general examination including head magnetic resonance imaging (MRI), abdominal ultrasound, chest CT, and whole-body bone scan; (III) patients who had complete tumor resection, angioplasty or vascular graft transplantation, chest wall reconstruction, partial lobectomy or lobectomy; (IV) patients with complete medical records and follow-up data.

Exclusion criteria included: (I) patients with tumor metastasis; (II) patients with multiple tumors; (III) lung and esophageal tumors; (IV) patients who were diagnosed as malignant lymphoma by puncture biopsy and were treated with radiotherapy or chemotherapy; $(\mathrm{V})$ patients with poor physical condition or other diseases that cannot tolerate the operation; (VI) patients who are reluctant to undergo surgical treatment.

\section{Patients' clinical data}

Thirty-four patients with complicated thoracic tumors admitted to our hospital from March 2016 to June 2019 and underwent radical tumor surgery were selected as the study subjects. These patients were randomly divided into the research group $(\mathrm{n}=17)$ in which the $3 \mathrm{D}$ printing technique was applied and control group $(\mathrm{n}=17)$ in which $3 \mathrm{D}$ printing technique was not applied. There were 11 males and 6 females in the research group, aged between 14 and 69 years with an average of $40.4 \pm 16.2$ years. Among these patients, there were 6 cases of giant thoracic tumors (tumor size $>$ one-half of the thoracic cavity), 4 cases of tumors invading the superior vena cava, 2 cases of tumors located on the top of the chest, and 5 cases of tumors invading other organs (including chest wall, lung lobe, diaphragm, or pericardium). In the control group, there were 12 males and 5 females, aged between 13 and 59 years with an average age of $42.7 \pm 14.2$ years. Among these patients, there were 7 cases of giant thoracic tumors, 5 cases of tumors invading blood vessels or trachea, 2 cases of tumors located on the top of the chest, and 3 cases of tumors invading the chest wall or lung lobes. There was no significant difference in gender, age, and tumor type between the research group and the control group (Table 1).

\section{$3 D$ digital modeling}

All patients underwent routine chest CT and wholebody bone emission computed tomography (ECT) before 
Table 1 Comparison of general information between the research group and the control group (mean \pm SD)

\begin{tabular}{|c|c|c|c|c|c|c|c|}
\hline Group & \multicolumn{2}{|c|}{ Gender } & Age (years) & \multicolumn{4}{|c|}{ Tumor type } \\
\hline Research group & 11 & 6 & $40.4+16.2$ & 6 & 4 & 2 & 5 \\
\hline Control group & 12 & 5 & $42.7+14.2$ & 7 & 5 & 2 & 3 \\
\hline
\end{tabular}

*, including chest wall, lung lobe, diaphragm, pericardium. SD, standard deviation.

surgery. The CT images were segmented and reconstructed three-dimensionally using the Amira software for thoracic tumors, pericardium, lungs, vertebrae, aorta, pulmonary artery, subclavian artery, brachiocephalic vein and superior vena cava. The $3 \mathrm{D}$ model was smoothened and simplified by Amira software and 4D Cinema software. The tumor volume, lung volume, and the distance from the tumor to important organs such as blood vessels and pericardium were measured.

\section{Preparation of 3D-PDF documents}

Complete 3D model data was imported into Deep Exploration 6.3, and model tree was edited, classified and renamed. File in U3D format was exported and the size of model file was controlled by setting the parameters in "Common" in the export interface. Acrobat Pro DC software was used to create a new PDF document, and U3D file was imported to the PDF document by using the "Add 3D" command in the "Rich Media" tool, generating a $3 \mathrm{D}$ view window with background being adjusted to white by "Background Color" tool. Finally, thoracic tumor and its adjacent structures field of view was adjusted and saved as the corresponding view file. PDF editing tool was used to design the page and saved to PDF format, creating a $3 \mathrm{D}-\mathrm{PDF}$ document with a $3 \mathrm{D}$ view. The operator can click on any structure through Acrobat or Adobe Reader software, and can freely move, rotate, stretch and translucently manipulate the $3 \mathrm{D}$ model of anatomical structure.

\section{$3 D$ printing}

According to the reconstructed 3D model, the stl format file was exported, and imported to the photosensitive resin $3 \mathrm{D}$ printer. A ratio of 2:1 3D printing was performed to obtain high-precision photosensitive resin $3 \mathrm{D}$ printed model, in which the tumor and lung are translucently printed, and the surgeons can observe the deep structures clearly. After printing, supportive structure was removed and the surface was smoothened and colored. The time for $3 \mathrm{D}$ printing and removal of supporting structures was approximately 2 days and the cost were approximately 1,400-2,000 dollars. The distance between the tumor and adjacent blood vessels and the pericardium was measured on a $3 \mathrm{D}$-printed model, and the simulated resection was performed to determine the optimal surgical plan.

\section{Development of surgical plan}

Preoperative evaluation was conducted based on $3 \mathrm{D}$ digital and printed models. Tumor locations, sizes, and the close relationship with anatomical spaces were closely determined to select the best surgical incision route and develop a preoperative surgical plan. Accurate thoracic tumor resection was simulated. During the operation, the tumor is completely removed to reduce the damage to the surrounding organs and blood vessels. All operations were performed by two thoracic surgeons. The study was conducted in accordance with the Declaration of Helsinki (as revised in 2013). The study was approved by the Ethical Committee of Southwest Hospital (IRB number: KY201846). Written informed consent was obtained from the patient for publication of this study and any accompanying images. A copy of the written consent is available for review by the editor-in-chief of this journal.

\section{Observation of parameters}

Intraoperative and postoperative indicators were compared between the two groups of patients. Intraoperative indicators included the time of surgery, occurrence in changes of surgical approach, and intraoperative blood loss. Postoperative indicators included the length of 
hospitalization, and the incidence of postoperative complications (such as wound bleeding, respiratory failure, relapsing pulmonary edema, arrhythmia, lung infections). A 6-month follow-up was performed, and the postoperative recurrence was compared between the two groups.

\section{Comparison between resectable and unresectable groups}

The above 34 patients who were treated by radical surgery were set as the resectable group. In the same period from March 2016 to June 2019, additional 12 cases of unresectable complicated thoracic tumors were selected as the unresectable group. 3D reconstruction was performed for all cases. Some imaging features and pathological malignancy of the tumor were scored according to the following standards and compared.

Scoring criteria: (I) location: lower chest: 1, middle chest: 2 , upper chest: 3 ; (II) size: $<1 / 3$ thoracic volume: 1 , 1/3-2/3 thoracic volume: $2,>2 / 3$ thoracic volume: 3 ; (III) smoothness of tumor-vascular contact surface: smooth: 1 , rough: 2, filling defect: 3; (IV) close contact with trachea, chest wall, pericardium, and diaphragm (1 point for each invasion); (V) pathological malignancy: low: 1, medium: 2, high: 3.

\section{Statistical analysis}

The data were statistically analyzed using SPSS25.0 (IBM, USA) software. Data were expressed as the frequency and percentage for categorical variables and the mean \pm standard deviation (SD) for continuous variables. The values are presented as the mean $\pm \mathrm{SD}$, the median and interquartile range (IQR). Comparison between the two groups was performed using Student's $t$-test and Mann-Whitney U test. To compare the proportions of patients, $\chi^{2}$ test or Fisher exact test was performed. All statistical tests were twosided and significance was defined as $\mathrm{P}<0.05$. There was no missing data.

\section{Results}

\section{$3 D$ digital and printed models of complicated thoracic tumors}

We created 3D digital and printed models of complicated thoracic tumors, and accurately formulated surgical plans. The classic cases are shown in Table 2 and Figures 1-4.

\section{Comparison of intraoperative indicators and hospitalization time between research and control group}

Operation time, the occurrence of changing surgical incision route, and bleeding volume in the research group were significantly lower than those in the control group $(\mathrm{P}<0.05)$. There was no significant difference in the hospitalization time between the two groups $(\mathrm{P}>0.05)$ (Table 3).

\section{Follow-up}

All patients were followed up for 6 months with one patient being lost during follow-up. Chest CT examination was performed for 3-6 months. There were no deaths, tumor recurrence or metastasis in the two groups.

\section{Comparison between resectable and unresectable groups}

The scores of tumor location, smoothness of tumorvascular contact surface and pathological malignancy in the unresectable group were significantly higher than those in the resectable group $(\mathrm{P}<0.05)$. There was no significant difference in the scores of tumor size and close contact with adjacent organs between the two groups. There was no significant difference in the scores of pathological malignancy between the $3 \mathrm{D}$ research group and control group $(\mathrm{P}>0.05)($ Tables 4,5$)$.

\section{Discussion}

Surgical resection plays an irreplaceable role in the treatment of thoracic tumors including embolization, radiotherapy, chemotherapy, etc. (10). For complicated thoracic tumors, it is difficult to evaluate the risk of operation before operation because of its large volume or compression or invasion to other tissues and organs. Furthermore, it is easy to damage important organs during surgery, leading to complications such as major bleeding and even death. The surgical treatment is difficult and the risk has increased significantly $(11,12)$. Therefore, the clinical treatment of complicated thoracic tumors is mostly based on thoracotomy, and surgical treatment remains to be a challenge (13). Preoperative evaluation and the formulation of a reasonable surgical plan based on comprehensive consideration of the location, nature of the tumors, the adjacent organs that it may invade, and 


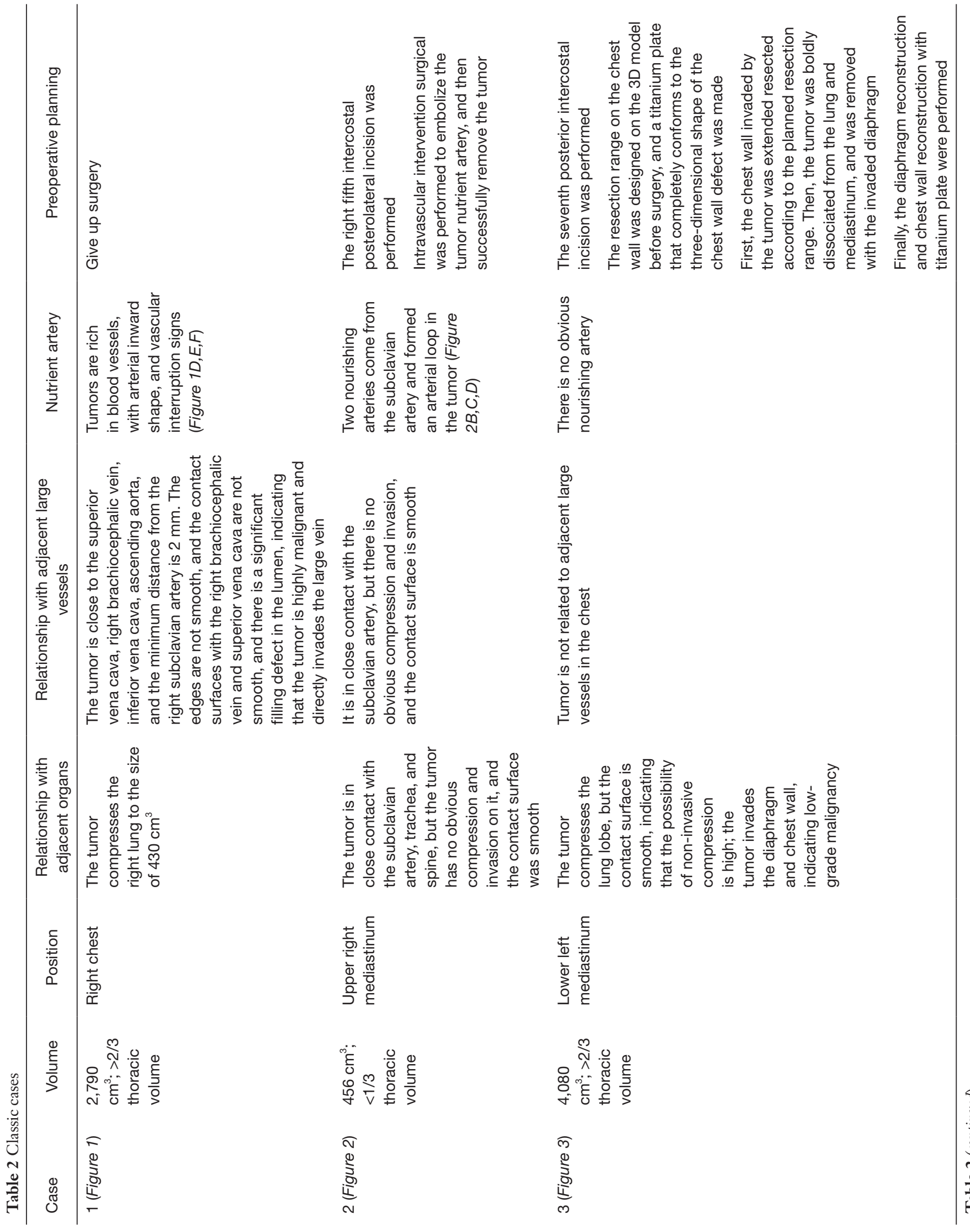




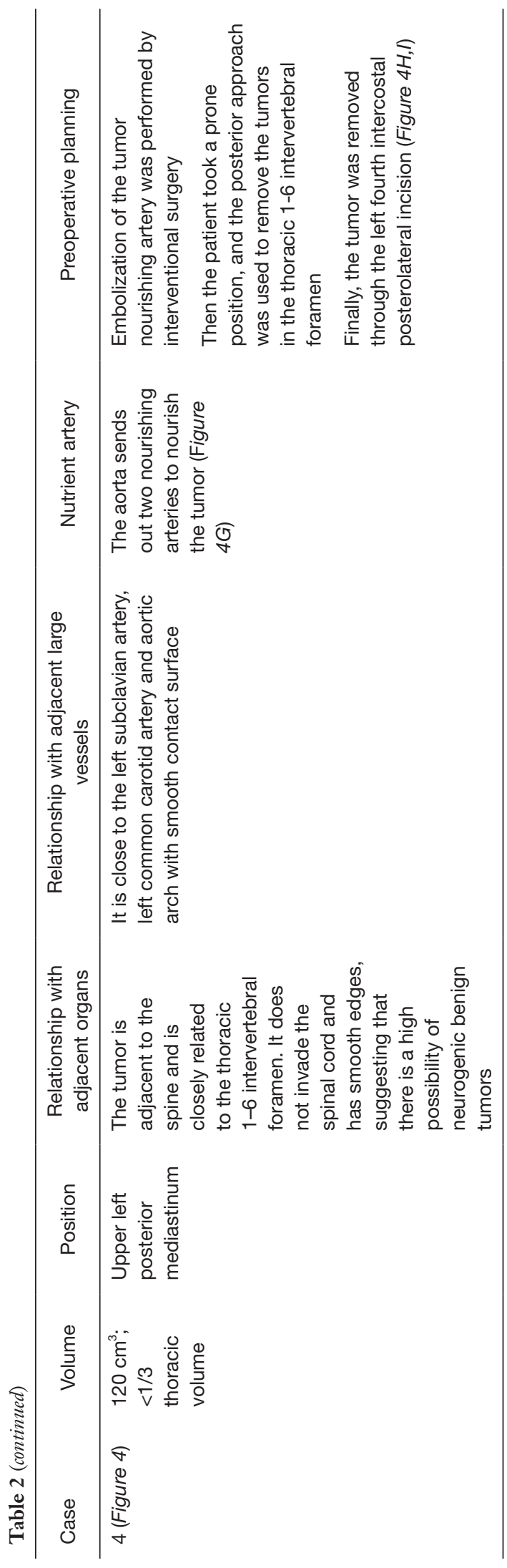

the existence of complications are the keys to ensuring the efficacy of surgery and improving the quality of life of patients.

The application of 3D printing in medical treatment has been increased rapidly. With the maturity of $3 \mathrm{D}$ printing technology, it has also been widely used in the medical field (14-16). At present, 3D printing technology has played a significant role in medical fields, e.g., surgical and preoperative planning, especially in orthopedics, traumatology or maxillofacial surgery $(17,18)$. Compared with traditional image, $3 \mathrm{D}$ printed solid models show the anatomy of the tumor more intuitively, adequately and clearly, providing a platform for the surgeon to determine the surgical plan, surgical incision route and intraoperative guidance, and can effectively evaluate the potential risks of surgery. By practicing the operation steps on the physical model and previewing the risk events of the operation, solutions could be proposed to ensure the success of the operation. In this study, $3 \mathrm{D}$ printing was used to make physical models for patients with complicated thoracic tumors, which effectively assisted surgeons to formulate safe and reliable surgical plans, reduce intraoperative complications and secondary damages, and achieve precise and personalized treatment. In addition, the surgeon can also use this $3 \mathrm{D}$ printed model to explain the individual situation of the chest tumor to the patient and the patient's family in detail, so that the patient and the family can more intuitively understand the condition, the necessity and risk of the operation, decrease the misunderstanding and increase the trust between the surgeon and the patients (19).

Application of $3 \mathrm{D}$ printing in complicated thoracic tumor surgery plans has been rarely reported $(20,21)$. In this study, we used 3D printing technology to perform preoperative evaluation of complicated thoracic tumors. For patients with giant thoracic tumors, we used 3D technology to evaluate the relationship between tumors and adjacent organs and blood vessels in advance to design the optimal surgical route and approach. For tumors invading blood vessels (subclavian artery, brachiocephalic vein, superior vena cava), we use $3 \mathrm{D}$ technology to accurately assess the 3D spatial relationship between tumors and large blood vessels, the extent of invasion, and the condition of lateral branches, which is conducive to the rapid implementation of accurate tumor resection and vascular reconstruction during the operation. For tumors located on the top of the chest, we used 3D technology to acknowledge the 3D spatial relationship between the tumor and the adjacent subclavian blood vessels, brachial plexus, and nutrient artery, design 

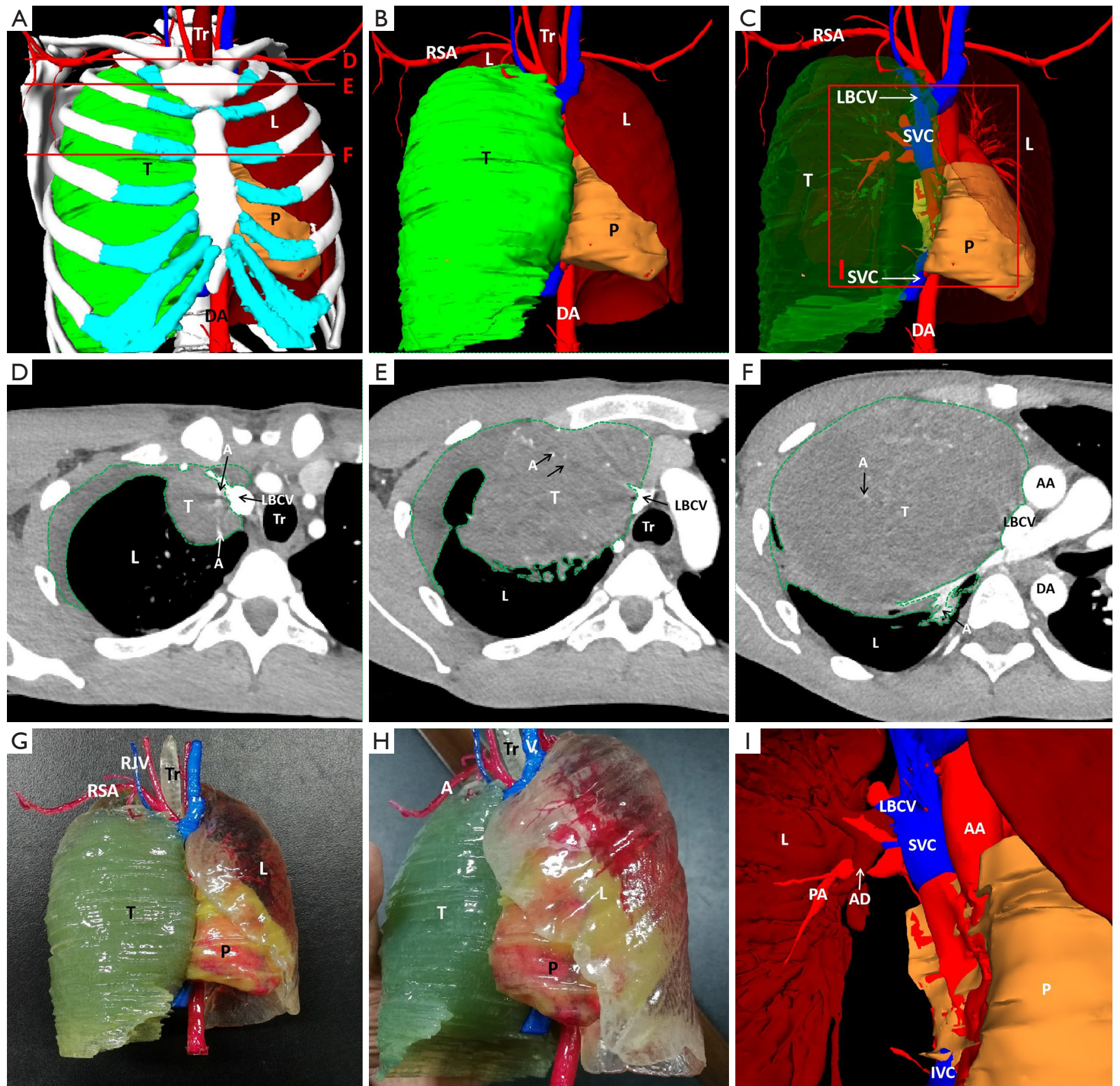

Figure $13 \mathrm{D}$ reconstructed images, CT transverse image and 3D printing model of giant thoracic tumor and its adjacent structures. (A,B,C) anterior view of 3D reconstructed images of giant thoracic tumor; (D,E,F) transverse CT images of giant thoracic tumor and its adjacent structures; $(\mathrm{G})$ anterior view of 3D printing model; $(\mathrm{H})$ antero-left view of 3D printing model; (I) magnification internal view of the interface of the tumor and the surrounding structures. 3D, three-dimensional; CT, computed tomography. Tr, trachea; T, tumor; L, lung; P, pericardium; DA, descending aorta; RSA, right subclavian artery; LBCV, left brachiocephalic vein; SVC, superior vena cava; A, artery; V, vein; AA, ascending aorta; RJV, right jugular vein; AD, arterial disconnection; PA, pulmonary artery; IVC, inferior vena cava. 

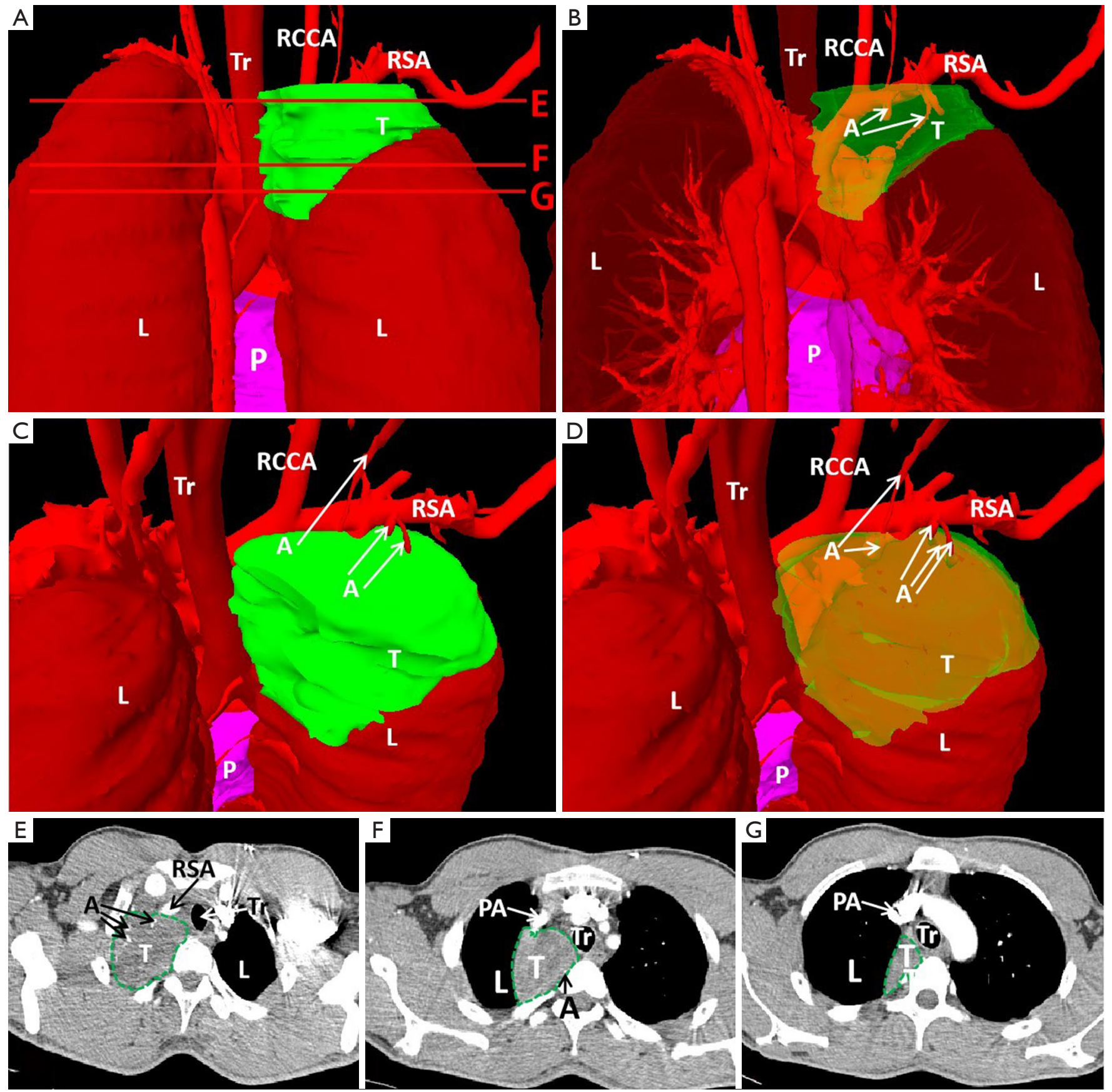

Figure 2 3D reconstructed images and CT transverse images of right-superior thoracic tumor and its adjacent structures. (A,B) Posterior view of $3 \mathrm{D}$ reconstructed images of right-superior thoracic tumor; (C,D) posterosuperior view of 3D reconstructed images of right-superior thoracic tumor; (E,F,G) transverse CT images of left-superior thoracic tumor and its adjacent structures. 3D, three dimensional; CT, computed tomography; RCCA, right common carotid artery; Tr, trachea; RSA, right subclavian artery; T, tumor; L, lung; P, pericardium; A, artery; PA, pulmonary artery. 

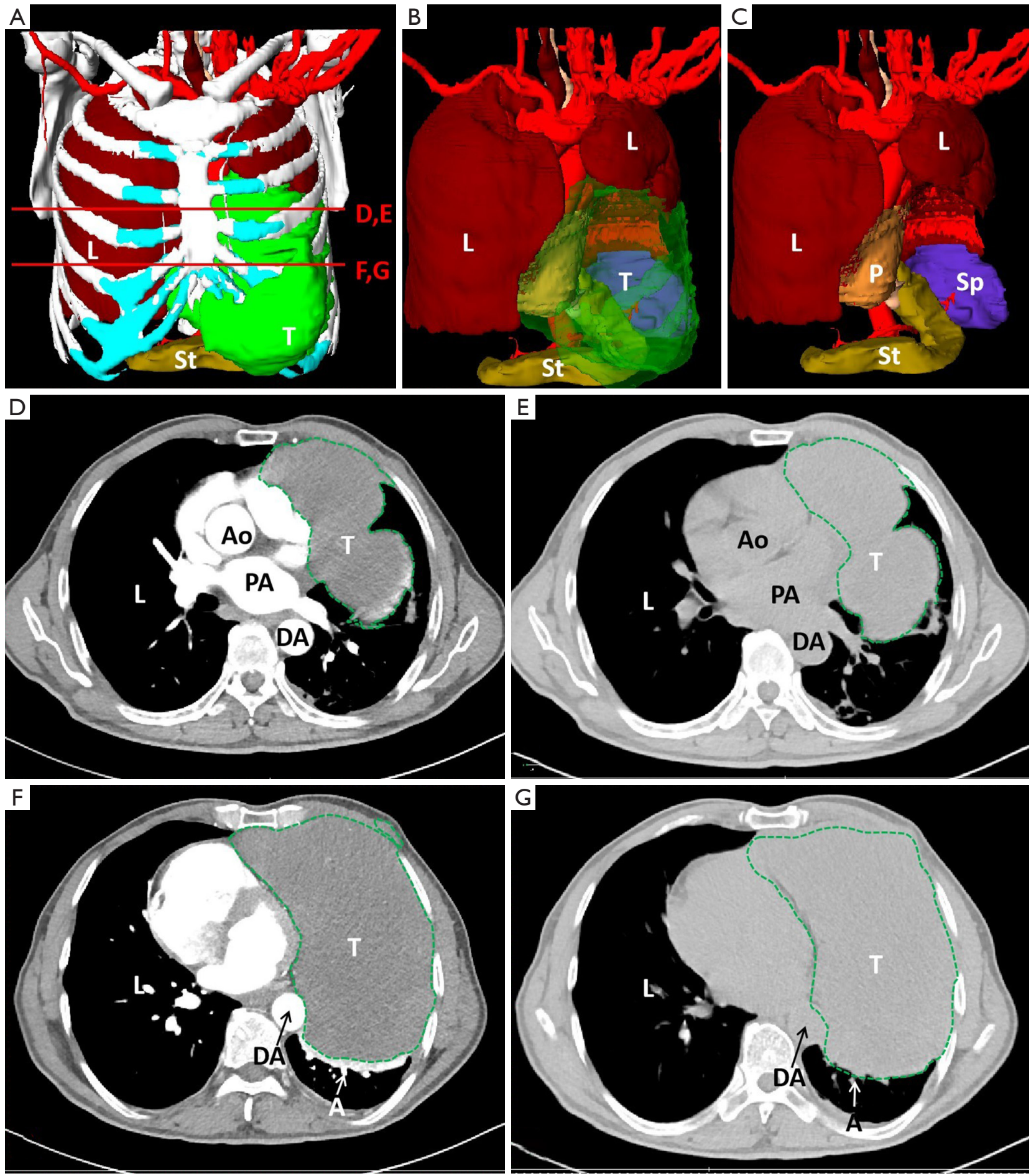

Figure 3 3D reconstructed images and CT transverse images of left-inferior giant thoracic tumor and its adjacent structures. $(A, B, C)$ Anterior view of 3D reconstructed images of left-inferior thoracic tumor; $(\mathrm{D}, \mathrm{F})$ transverse $\mathrm{CTA}$ images of left-inferior thoracic tumor; $(\mathrm{E}, \mathrm{G})$ transverse plain CT images of left-inferior thoracic tumor. 3D, three dimensional; CT, computed tomography; CTA, computed tomography angiography; ST, stomach; T, tumor; L, lung; P, pericardium; Sp, spleen; Ao, aorta; PA, pulmonary artery; DA, descending aorta; Ao, aorta; PA, pulmonary artery; A, artery. 

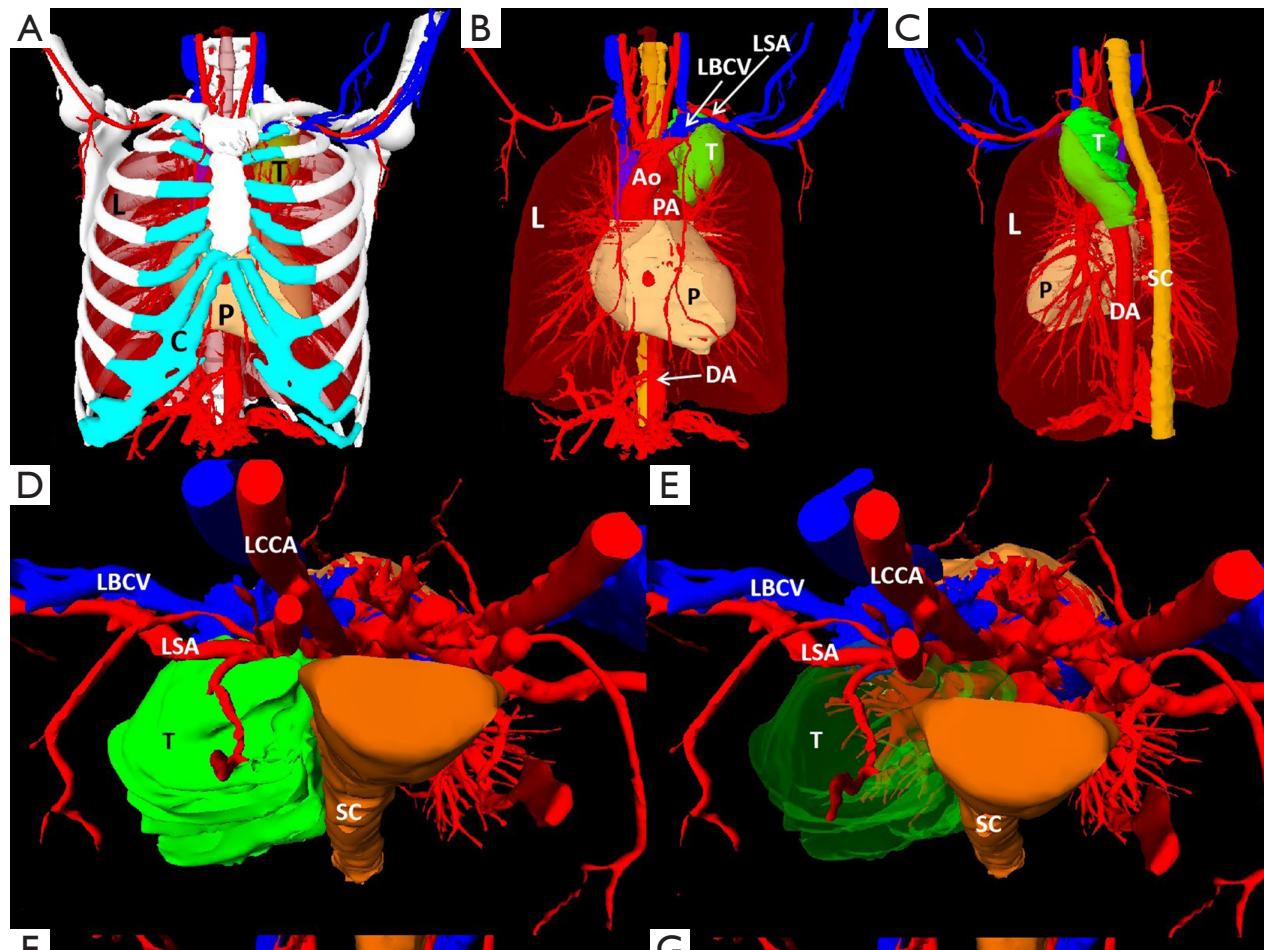

\section{$\mathrm{E}$}

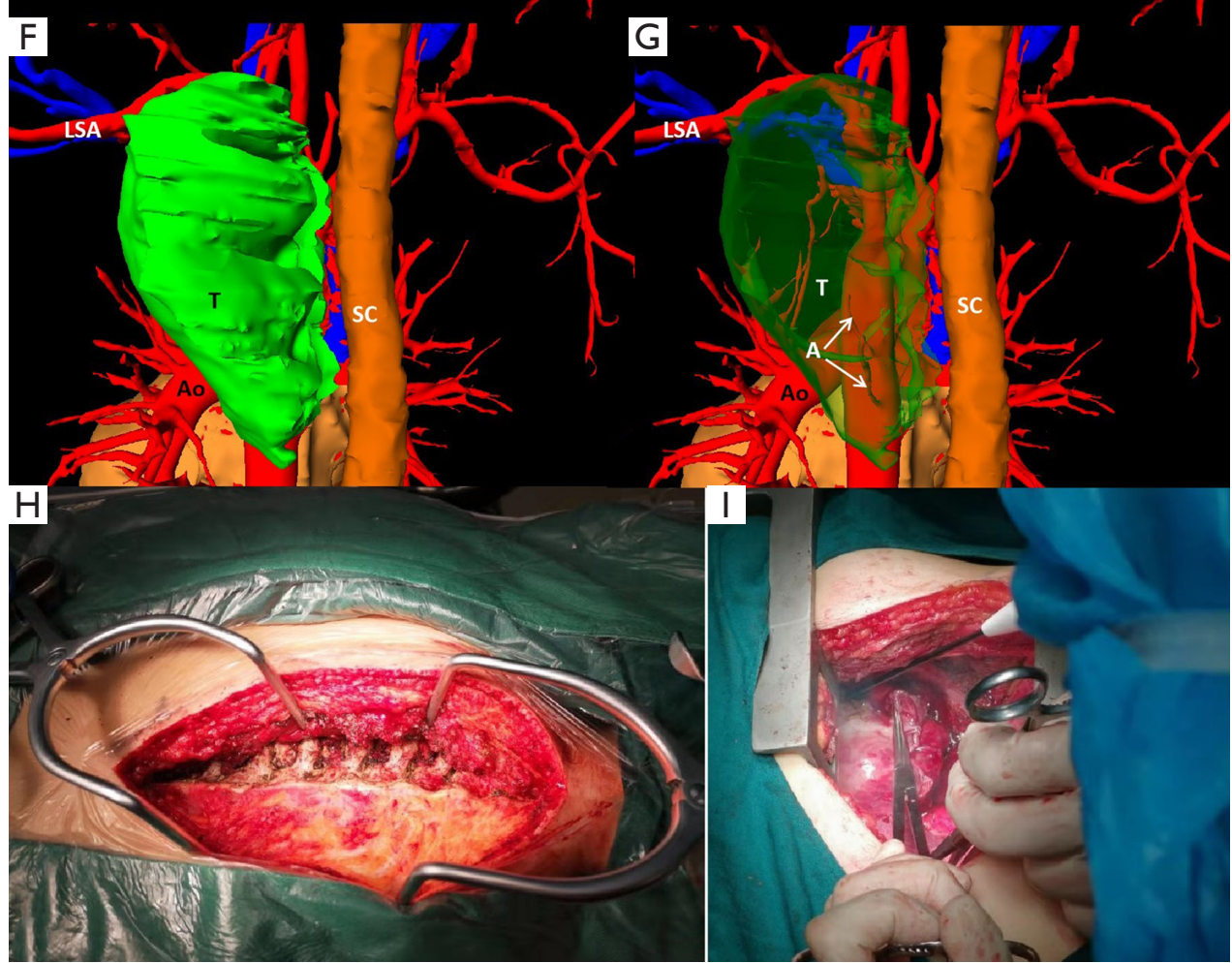

Figure 4 3D reconstructed images and CT transverse images of left-superior-posterior thoracic tumor and its adjacent structures. (A,B,C) Anterior view of 3D reconstructed images of left-superior-posterior thoracic tumor; (D) superior-posterior view of left-superior-posterior thoracic tumor; (E) superior-posterior view of left-superior-posterior thoracic tumor with tumor transparent; (F) left view of left-superiorposterior thoracic tumor; $(\mathrm{G})$ left view of left-superior-posterior thoracic tumor with tumor transparent; $(\mathrm{H})$ spinal surgery; (I) thoracic surgery. 3D, three dimensional; CT, computed tomography; T, tumor; L, lung; P, pericardium; LBCV, left brachiocephalic vein; LSA, left subclavian artery; Ao, aorta; PA, pulmonary artery; DA, descending aorta; SC, spinal cord; LCCA, left common carotid artery; A, artery. 
Table 3 Comparison of the intraoperative variables, incidence of complications and hospitalization time between the two groups of patients

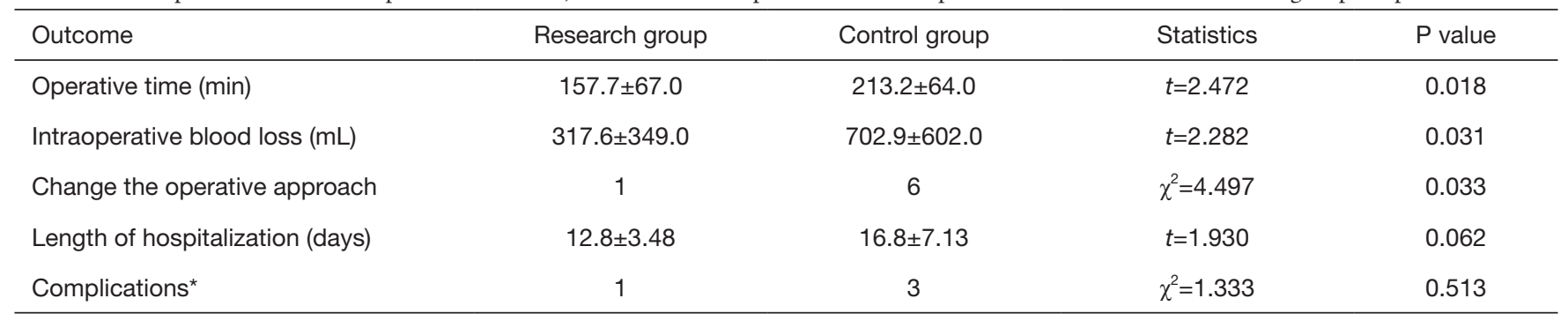

Values are presented as median (range) or mean \pm standard deviation (SD). *, including bleeding, respiratory failure, relapsing pulmonary edema, lung infections.

Table 4 Comparison between resectable and unresectable groups

\begin{tabular}{lcrr}
\hline Variables & Resectable group $(\mathrm{n}=34)$ & Unresectable groups $(\mathrm{n}=12)$ & $\mathrm{Z}$ \\
\hline Size & $1.88(1.00-2.00)$ & $1.92(1.00-2.75)$ & -0.109 \\
Location & $2.00(1.00-3.00)$ & $3.00(3.00-3.00)$ & -4.056 \\
$\begin{array}{l}\text { Smoothness of tumor-vascular contact } \\
\text { surface }\end{array}$ & $1.71(1.00-3.00)$ & $2.67(2.00-3.00)$ & -3.142 \\
$\begin{array}{l}\text { Close contact with trachea, chest wall, } \\
\text { pericardium, and diaphragm }\end{array}$ & $2.18(2.00-3.00)$ & $1.92(1.00-2.76)$ & 0.000 \\
Pathological malignancy & & & -1.083 \\
\hline
\end{tabular}

Values are presented as median (range).

Table 5 Comparison of pathological malignancy between two groups

\begin{tabular}{|c|c|c|c|c|c|c|c|c|c|c|c|c|c|}
\hline Group & \multicolumn{5}{|c|}{ Low } & \multicolumn{3}{|c|}{ Medium } & \multicolumn{5}{|c|}{ High } \\
\hline \multicolumn{14}{|l|}{$\begin{array}{l}\text { Resectable } \\
\text { group }\end{array}$} \\
\hline $\begin{array}{l}\text { Control } \\
\text { group * }\end{array}$ & 0 & 1 & 4 & 1 & 1 & 3 & 2 & 0 & 3 & 2 & 0 & 0 & 0 \\
\hline $\begin{array}{l}\text { Unresectable } \\
\text { groups }^{\star \star}\end{array}$ & - & 0 & 0 & 0 & 0 & 0 & 0 & 0 & 2 & 2 & 3 & 1 & 4 \\
\hline
\end{tabular}

*, research group (3D) vs. control group, $\mathrm{P}=0.685 ;{ }^{* *}$, resectable group vs. unresectable groups, $\mathrm{P}=0.000$.

the optimal surgical approach, and embolism the nutrient artery in advance. For tumors that invade other organs (chest wall, lung, diaphragm, pericardium), we use 3D technology to acknowledge in advance the spatial relationship between the tumor and the invading spatial organs, the scope of the invasion, and design a $3 \mathrm{D}$ shape of the substitute. In this study, we showed that the average operation time, intraoperative blood loss, and incidence of changes in surgical incisions in the research group were significantly reduced compared with the control group. In addition, the occurrence of complications is often the main cause of surgical failure. Postoperative complications of complicated 
thoracic tumor surgery mainly include recurrent pulmonary edema, pulmonary infection, arrhythmia, respiratory failure, pleural effusion, and wound bleeding $(22,23)$. The incidence of total complications was no significant difference between the research group and the control group. At the same time, we showed that there was no tumor recurrence or metastasis in both groups, indicating that the $3 \mathrm{D}$ printing technology did not affect the surgical efficacy.

We compared these resectable cases with some unresectable cases in the same period, and tried to score and compare some factors that may affect the resectability. The results showed that the location of the tumor in the thoracic cavity and the smoothness of the contact surface between the tumor and the adjacent large blood vessels may be the important factors affecting the removal of the tumor. Further analysis showed that the unresectable tumors were located at the top of the chest, and all of them had invasion to the adjacent subclavian artery or vein, superior vena cava, even the brachial plexus and trachea (the score of the contact surface between the tumor and the adjacent large vessels was $2-3$ ), which may be the main reason why the operation could not be performed. The tumor invading the superior vena cava, the chest wall, pericardium, or the diaphragm is not the reason of non resectability, because these structures invaded by the tumor can be reconstructed.

In summary, 3D modeling and printing technology has been frequently applied in assisting and optimizing surgery. For complicated thoracic tumors, it can effectively help patients receive accurate treatment, reduce the operation time and bleeding, reduce the risk of surgery, facilitate the patient's postoperative recovery, shorten the length of hospitalization and greatly reduces the medical expenses. The limitation of this study lies in the small number of cases, lack of the evaluation on the long-term efficacy and prognosis of patients, and the lack of comparison of patients' treatment compliance. We expect to increase the number of surgical procedures in the future to improve $3 \mathrm{D}$ printing in assisting thoracic tumor resection and to develop criteria for the procedure.

\section{Acknowledgments}

Funding: This work was supported by the National Natural Science Foundation of China (No. 31971113 and 31671251; http://www. nsfc.gov.cn/), National Key Research and Development Program of China (No. 2016YFC0106400); Postgraduate Teaching Reform Project in Chongqing (No. yjg183144; http://www.cqjw. gov.cn/),
Chongqing basic research and frontier exploration project (No. cstc2018jcyjAX0537), University Funded Science and Technology Innovation Capacity Improvement Project (No. 2019XYY14).

\section{Footnote}

Reporting Checklist: The authors have completed the STROBE reporting checklist. Available at http://dx.doi. org/10.21037/atm-20-1791

Data Sharing Statement: Available at http://dx.doi. org/10.21037/atm-20-1791

Peer Review File: Available at http://dx.doi.org/10.21037/ atm-20-1791

Conflicts of Interest: All authors have completed the ICMJE uniform disclosure form (available at http://dx.doi. org/10.21037/atm-20-1791). The authors have no conflicts of interest to declare.

Ethical Statement: The authors are accountable for all aspects of the work in ensuring that questions related to the accuracy or integrity of any part of the work are appropriately investigated and resolved. The study was conducted in accordance with the Declaration of Helsinki (as revised in 2013). The study was approved by the Ethical Committee of Southwest Hospital (IRB number: KY201846). Written informed consent was obtained from the patient for publication of this study and any accompanying images. A copy of the written consent is available for review by the editor-in-chief of this journal.

Open Access Statement: This is an Open Access article distributed in accordance with the Creative Commons Attribution-NonCommercial-NoDerivs 4.0 International License (CC BY-NC-ND 4.0), which permits the noncommercial replication and distribution of the article with the strict proviso that no changes or edits are made and the original work is properly cited (including links to both the formal publication through the relevant DOI and the license). See: https://creativecommons.org/licenses/by-nc-nd/4.0/.

\section{References}

1. Wu Y, Luo N, Tan L, et al. Three-dimensional reconstruction of thoracic structures: based on 
Chinese Visible Human. Comput Math Methods Med 2013;2013:795650.

2. Kurenov SN, Ionita C, Sammons D, et al. Threedimensional printing to facilitate anatomic study, device development, simulation, and planning in thoracic surgery. J Thorac Cardiovasc Surg 2015;149:973-9.e1.

3. Wei XB, Xu J, Li N, et al. The role of three-dimensional imaging in optimizing diagnosis, classification and surgical treatment of hepatocellular carcinoma with portal vein tumor thrombus. HPB(Oxford) 2016;18:287-95.

4. Anderson PA. Clinical applications of $3 \mathrm{D}$ printing. Spine (Phila Pa 1976) 2017;42 Suppl 7:S30-S31.

5. Cai H. Application of $3 \mathrm{D}$ printing in orthopedics: status quo and opportunities in China. Ann Transl Med 2015;3:S12.

6. Chao I, Young J, Coles-Black J, et al. The application of three-dimensional printing technology in anaesthesia: a systematic review. Anaesthesia 2017;72:641-50.

7. $\mathrm{Wu} \mathrm{Y,} \mathrm{Chen} \mathrm{N}, \mathrm{Xu} \mathrm{Z}$, et al. Application of $3 \mathrm{D}$ printing technology to thoracic wall tumor resection and thoracic wall reconstruction. Journal of Thoracic Disease. J Thorac Dis 2018;10:6880-90.

8. Zhang X, Xu Z, Tan L, et al. Application of threedimensional reconstruction and printing as an elective course for undergraduate medical students: an exploratory trial. Surg Radiol Anat 2019;41:1193-204.

9. Javan R, Schickel M, Zhao YL, et al. Using 3D-Printed Mesh-LikeBrain Cortex with Deep Structures for Planning Intracranial EEG Electrode Placement. J Digit Imaging 2020;33:324-33.

10. Duwe BV, Sterman DH, Musani AI. Tumors of the mediastinum. Chest 2005;128:2893-909.

11. Wu CF, Gonzalez-Rivas D, Wen CT, et al. Single-port video-assisted thoracoscopic mediastinal tumour resection. Interact Cardiovasc Thorac Surg 2015;21:644-9.

12. Motus IY, Bazhenov AV, Massard G. Surgery for huge mediastinal tumors. Asian Cardiovasc Thorac Ann 2015;23:846-50.

Cite this article as: Tan D, Yao J, Hua X, Li J, Xu Z, Wu Y, $\mathrm{Wu} \mathrm{W}$. Application of 3D modeling and printing technology in accurate resection of complicated thoracic tumors. Ann Transl Med 2020;8(21):1342. doi: 10.21037/atm-20-1791
13. Ishibashi H, Ishikawa $\mathrm{Y}$, Asakawa A, et al. Chylothorax post thoracoscopic surgery for an anterior mediastinal tumor. Gen Thorac Cardiovasc Surg 2018;66:372-5.

14. Srougi V, Rocha BA, Tanno FY, et al. The use of threedimensional printers for partial adrenalectomy: estimating the resection limits. Urology 2016;90:217-20.

15. Zhang Y, Ge HW, Li NC, et al. Evaluation of threedimensional printing for laparoscopic partial nephrectomy of renal tumors: a preliminary report. World J Urol 2016;34:533-7.

16. AlAli AB, Griffin MF, Butler PE. Three-Dimensional Printing Surgical Applications. Eplasty 2015;15:e37.

17. Martelli N, Serrano C, van den Brink H, et al. Advantages and disadvantages of 3-dimensional printing in surgery: a systematic review. Surgery 2016;159:1485-500.

18. Langridge B, Momin S, Coumbe B, et al. Systematic review of the use of 3-dimensional printing in surgical teaching and assessment. J Surg Educ 2018;75:209-21.

19. Girón-Vallejo Ó, García-Calderón D, Ruiz-Pruneda R, et al. Three- dimensional printed model of bilateral Wilms tumor: A useful tool for planning nephron sparing surgery. Pediatr Blood Cancer 2018. doi: 10.1002/pbc.26894.

20. George E, Barile M, Tang A, et al. Utility and Reproducibility of 3-Dimensional Printed Models in PreOperative Planning of Complex Thoracic Tumors. J Surg Oncol 2017;116:407-15.

21. Gillaspie EA, Matsumoto JS, Morris NE, et al. From 3-Dimensional Printing to 5-Dimensional Printing: Enhancing Thoracic Surgical Planning and Resection of Complex Tumors. Ann Thorac Surg 2016;101:1958-62.

22. Wang KR, Liu FF, Zhou YF. Difficulties in tracheal extubation due to phrenic nerve injury during massive mediastinal tumor resection: A case report. Medicine (Baltimore) 2019;98:e16252.

23. Liu Y, Lu T, Fan H, et al. Surgical Approaches to Nonthyrogenic and Non-thymic Mediastinal Tumors of the Thoracic Inlet. Thorac Cardiovasc Surg 2018;66:336-43. 